\title{
Kinetic modeling of anaerobic degradation of plant-derived aromatic mixtures by Rhodopseudomonas palustris
}

\author{
Yanjun Ma • Timothy J. Donohue $\cdot$ Daniel R. Noguera $\mathbb{B}$
}

Received: 1 December 2020/Accepted: 23 February 2021 / Published online: 6 March 2021

(C) The Author(s) 2021

\begin{abstract}
Rhodopseudomonas palustris is a model microorganism for studying the anaerobic metabolism of aromatic compounds. While it is well documented which aromatics can serve as sole organic carbon sources, co-metabolism of other aromatics is poorly understood. This study used kinetic modeling to analyze the simultaneous degradation of aromatic compounds present in corn stover hydrolysates and model the co-metabolism of aromatics not known to support growth of $R$. palustris as sole organic substrates. The simulation predicted that $p$-coumaroyl amide and feruloyl amide were hydrolyzed to $p$ coumaric acid and ferulic acid, respectively, and further transformed via $p$-coumaroyl-CoA and feruloyl-CoA. The modeling also suggested that
\end{abstract}

Supplementary Information The online version of this article (https://doi.org/10.1007/s10532-021-09932-3) contains supplementary material, which is available to authorized users.

Y. Ma · T. J. Donohue · D. R. Noguera $(\bowtie)$

Great Lakes Bioenergy Research Center, Wisconsin

Energy Institute, University of Wisconsin-Madison,

Madison, WI 53726, USA

e-mail:dnoguera@wisc.edu

T. J. Donohue

Department of Bacteriology, University of Wisconsin-

Madison, Madison, WI 53706, USA

D. R. Noguera

Department of Civil and Environmental Engineering, University of Wisconsin-Madison, Madison,

WI 53706, USA metabolism of $p$-hydroxyphenyl aromatics was slowed by substrate inhibition, whereas the transformation of guaiacyl aromatics was inhibited by their $p$ hydroxyphenyl counterparts. It also predicted that substrate channeling may occur during degradation of $p$-coumaroyl-CoA and feruloyl-CoA, resulting in no detectable accumulation of $p$-hydroxybenzaldehyde and vanillin, during the transformation of these CoA ligated compounds to $p$-hydroxybenzoic acid and vanillic acid, respectively. While the simulation correctly represented the known transformation of $p$ hydroxybenzoic acid via the benzoyl-CoA pathway, it also suggested co-metabolism of vanillic acid and syringic acid, which are known not to serve as photoheterotrophic growth substrate for $R$. palustris.

Keywords Kinetic modeling Rhodopseudomonas palustris Plant-derived aromatics - Anaerobic degradation $\cdot$ Co-metabolism $\cdot$ Substrate inhibition

\section{Introduction}

As the largest renewable feedstock comprising aromatics, lignin in lignocellulosic biomass is increasingly recognized as a great potential source of valuable industrial and commercial products (Ragauskas et al. 2014; Tuck et al. 2012). The structure of lignin is derived from three monomers differing in their degree 
of methoxylation ( $p$-coumaroyl, coniferyl, and sinapyl alcohols), which result in the corresponding $p$-hydrophenyl $(H)$, guaiacyl $(\mathrm{G})$, and syringyl (S) aromatic units in lignin (Davis et al. 2016; Wong 2009). Depolymerization of lignin generates highly heterogeneous mixtures of aromatic monomers, which makes it challenging for extracting and recovering individual products. Microbial treatment has been considered as a possible strategy to covert the heterogeneous mixtures of plant-derived aromatic to a few or even a single compound that can be recovered (Gall et al. 2017; Perez et al. 2019). We have a general interest in identifying microbial routes to transform plant-derived aromatic compounds by microorganisms that could be engineered to make single products from heterogeneous mixtures. The purple non-sulfur bacterium Rhodopseudomonas palustris is one of such model microorganisms, which is known to be metabolically versatile in utilizing aromatic compounds under anaerobic conditions (Austin et al. 2015).

The anaerobic metabolism of aromatic compounds by $R$. palustris has been extensively studied and demonstrated to occur via the benzoyl-CoA pathway (Egland et al. 1997; Gall et al. 2013; Harwood et al. 1998). In this pathway, one of several aromatic acids are activated by ligation of Coenzyme A (CoA), and then, the aromatic ring undergoes sequential reduction until cleavage, leading to further transformation to acetyl-CoA, which enters central metabolism. Benzoic and $p$-hydroxybenzoic acids can sustain anaerobic photoheterotrophic growth of $R$. palustris when provided as sole organic carbon sources. Other aromatic compounds known to support anaerobic $R$. palustris growth as sole organic substrates are initially transformed to benzoic acid, $p$-hydroxybenzoic acid, or to their CoA ligated forms. For instance, $p$ coumaric acid has been shown to be metabolized via the benzoyl-CoA pathway after enzymes in upper pathways convert $p$-coumaric to $p$-hydroxybenzoic acid (Hirakawa et al. 2012; Pan et al. 2008). In addition, cinnamic acid, 3-phenylpropionic acid, and 5-phenylvaleric acid have been shown to undergo Boxidation to benzoyl-CoA before entering the benzoyl-CoA pathway (Elder et al. 1992).

The existing literature on anaerobic degradation of aromatics by $R$, palustris shows that aromatic acids with substitutions other than a hydroxyl at the para position to the carboxylic group cannot serve as sole organic carbon sources for photoheterotrophic growth of $R$. palustris (Harwood and Gibson 1988). There is a recent report of an $R$. palustris strain evolved to degrade syringic acid, which does not use the benzoylCoA pathway (Oshlag et al. 2020). In addition, even though there is a limited number of aromatic compounds that support photoheterotrophic growth as sole organic carbon sources, $R$. palustris can metabolize a broad set of aromatic compounds when multiple aromatics are present (Austin et al. 2015; Gall et al. 2013). For instance, while it cannot be used as a sole organic carbon source, protocatechuic acid (3,4-dihydroxybenzoic acid) has been shown to be degraded via the benzoyl-CoA pathway if either benzoic acid or $p$ hydroxybenzoic acid is provided as a co-substrate (Gall et al. 2013). Furthermore, the ability of $R$. palustris to co-metabolize substrates that cannot serve as sole organic substrates for photoheterotrophic growth appears to be a feature not limited to aromatic compounds (Govindaraju et al. 2019).

We have previously reported on the degradation of multiple aromatic compounds when $R$. palustris is grown on aromatic-containing hydrolysates produced from lignocellulosic biomass (Austin et al. 2015). The most abundant aromatic compounds in these hydrolysates were $p$-coumaroyl amide and feruloyl amide, with 16 other aromatic compounds detected at lower concentrations (Austin et al. 2015). In these experiments, $R$. palustris utilized most of the aromatics in the hydrolysates with extracellular accumulation of $p$ hydroxybenzoic acid, vanillic acid, and protocatechuic acid. Since the ability of $R$. palustris to anaerobically degrade most of the aromatic compounds found in these hydrolysates has not been previously documented, either as sole substrates or cosubstrates, we used kinetic modeling to investigate $R$. palustris metabolism of these compounds in the batch experiments reported by Austin et al. (2015). The goal was to generate hypotheses of degradation pathways and factors that influence co-metabolism of aromatic compounds by $R$. palustris. These kinetic models propose pathways that are consistent with existing knowledge and best fit the trends of aromatic metabolism observed when this bacterium grows in the presence of these biomass hydrolysates. 


\section{Materials and methods}

Batch reactor

As described in Austin et al. (2015), the batch reactor contained $1000 \mathrm{~mL}$ of ammonia fiber expansion (AFEX) treated corn stover hydrolysates (ACSH) and was inoculated with $20 \mathrm{~mL}$ R. palustris CGA009 pregrown in minimal medium. The reactor was operated anaerobically for about $192 \mathrm{~h}$ at $28{ }^{\circ} \mathrm{C}$ and exposed to continuous light. Data for the batch experiment was obtained from Austin et al. (2015), including cell optical density measured at $600 \mathrm{~nm}$ $\left(\mathrm{OD}_{600}\right)$, concentration of aromatic compounds in the starting ACSH (Table 1 and Fig. S1) and over time.

Kinetic models

The rate of change of the concentration of each aromatic compound in the batch reactor is expressed as:

$\frac{d S}{d t}=r_{p} X-r_{s} X$

where $\mathrm{S}$ stands for substrate concentration $\left(\mu \mathrm{mol} \mathrm{l}^{-1}\right)$, $\mathrm{X}$ represents biomass concentration $\left(\mathrm{mg} \mathrm{l}^{-1}\right)$, and $\mathrm{r}_{\mathrm{p}}$ and $r_{s}$ stand for specific substrate production and consumption rates $\left(\mu \mathrm{mol} \mathrm{mg}^{-1} \mathrm{~h}^{-1}\right)$, respectively. Biomass concentrations were estimated from measured $\mathrm{OD}_{600}$ using a correlation determined in separate experiments. The Gompertz function (Gompertz 1825) was used to model $X$ in the batch reactor as a function of time (Fig. S8).

Substrate production and consumption rates were simulated using either first order rates (Eq. 2) or rates that account for inhibition by a secondary substrate (Eq. 3). In these equations, $k$ is the first order reaction rate $\left(1 \mathrm{mg}^{-1} \mathrm{~h}^{-1}\right), \mathrm{k}_{\mathrm{i}}$ is the inhibition factor $\left(\mu \mathrm{mol} \mathrm{l}^{-1}\right)$, $S_{i}$ is the concentration of inhibitory substrate $(\mu \mathrm{mol}$ $\left.1^{-1}\right)$, and all other terms are as defined above.

$r=k S$

$r=k\left(\frac{k_{i}}{k_{i}+S_{i}}\right) S$

The differential equations representing substrate concentration over time (Eq. 1) were solved numerically using Euler's method (Atkinson 1989) with a time step of $1 \mathrm{~h}$, and expressed as a user-defined function in $\mathrm{R}$ ( $\mathrm{R}$ Core Team 2019). The best-fit parameters $\mathrm{k}$ and $\mathrm{k}_{\mathrm{i}}$ were estimated by minimizing the Residual Sum of Squares (RSS) between the modeled and measured substrate concentrations using the optim function in R (R Core Team 2019).

\section{Results and discussion}

Proposed pathways for anaerobic co-metabolism of p-hydroxyphenyl, guaiacyl and syringyl type aromatics by $R$. palustris

The abundant aromatic compounds identified in the lignocellulosic biomass hydrolysates used by Austin et al. (2015) included $p$-hydroxyphenyl ( $\mathrm{H}$; no methoxy substitution), guaiacyl (G; with one metamethoxy substitution) and syringyl ( $\mathrm{S}$; with two metamethoxy substitutions) type aromatics (Table 1, Fig. S1) derived from pretreatment of corn stover using the ammonia fiber expansion (AFEX) pretreatment process (Teymouri et al. 2004). Among these compounds, it is well established that benzoic acid, $p$ hydroxybenzoic acid, $p$-coumaric acid, and protocatechuic acid are metabolized by $R$. palustris anaerobically through the benzoyl-CoA pathway (Egland et al. 1997; Gall et al. 2013; Harwood et al. 1998; Hirakawa et al. 2012; Pan et al. 2008). In contrast, pathways for the degradation of the most abundant aromatic compounds in the hydrolysate, $p$-coumaroyl amide and feruloyl amide, have not been elucidated.

To inform our modeling of aromatic co-metabolism, we propose here tentative pathways for $p$ coumaroyl amide and feruloyl amide degradation (Fig. 1) based on existing evidence and hypothetical reactions. Since these aromatic amides were completely transformed by R. palustris (Austin et al. 2015) and it is known that bacterial amidases can hydrolyze a broad range of amides (including aromatic amides) to produce the corresponding acids (Hirrlinger et al. 1996; Ismailsab et al. 2017; Ruan et al. 2016), we propose that $p$-coumaroyl amide and feruloyl amide are hydrolyzed by amidases to $p$-coumaric acid and ferulic acid, respectively (Fig. 1). Once deamidated, we propose that these aromatic acids can be subsequently activated by CoA ligation. Then, with an enoyl-CoA hydratase/aldolase, $p$-coumaroyl-CoA and feruloyl-CoA can be converted to the respective aldehydes $p$-hydroxybenzaldehyde and vanillin. Pan 


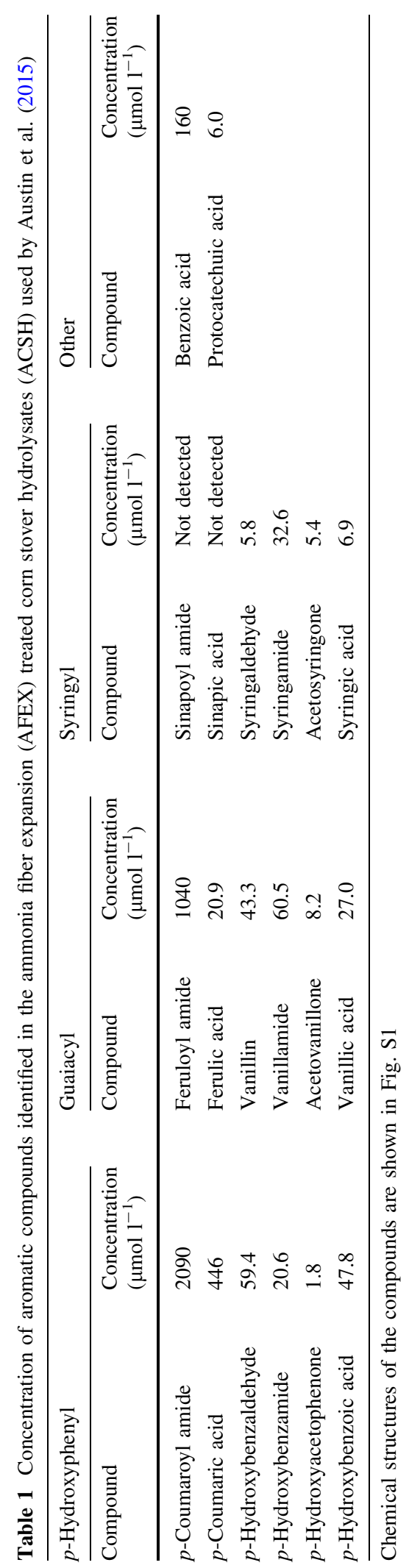




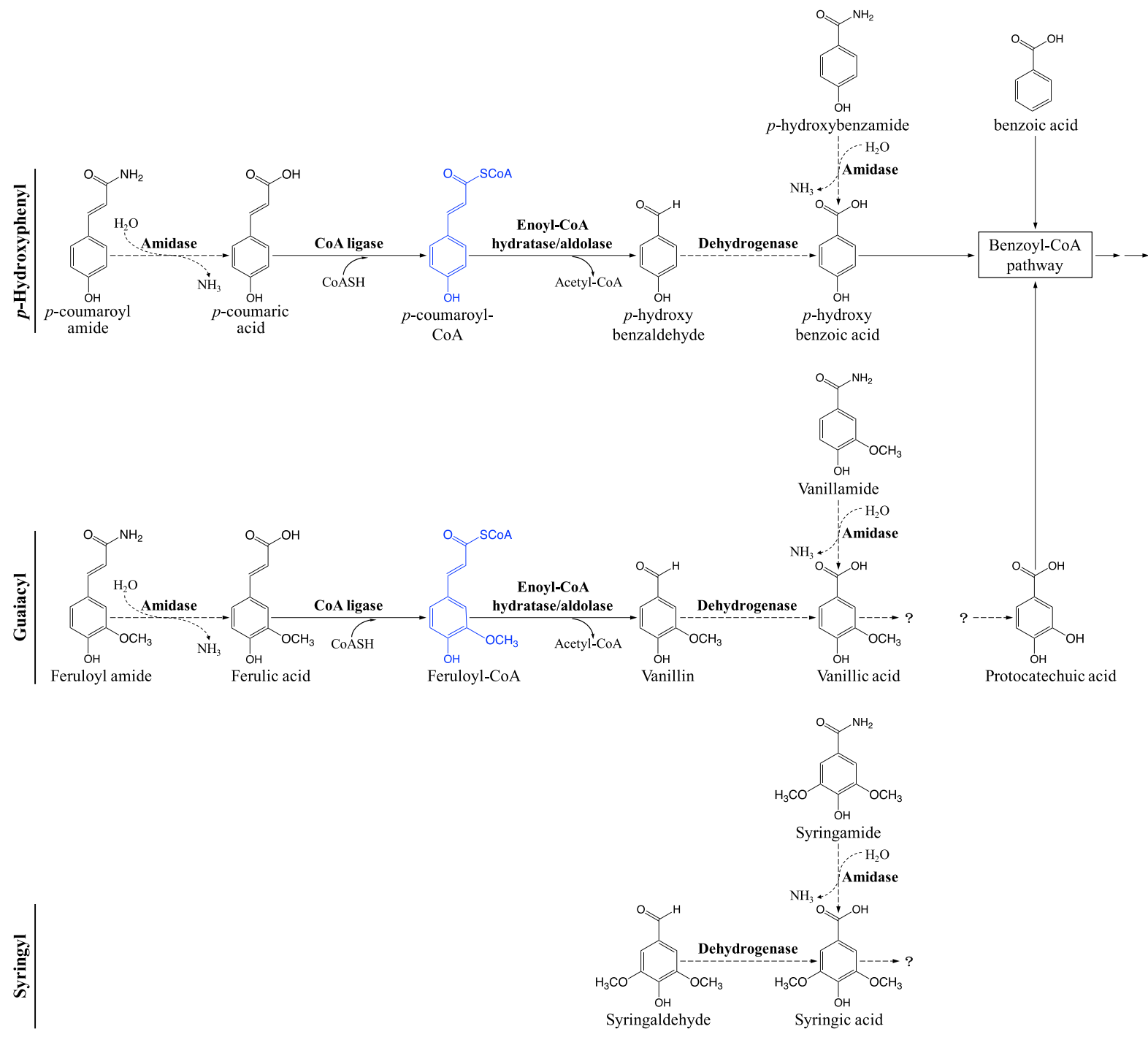

Fig. 1 Proposed degradation pathways for plant-derived aromatic compounds detected in the experiment of Austin et al. (2015). Solid arrows indicated reactions that are experimentally demonstrated in $R$. palustris. Dashed arrows

et al. (2008) described the CoA ligase (CouB) and enoyl-CoA hydratase/aldolase $(\mathrm{CouA})$ gene products that convert $p$-coumaric acid to $p$-hydroxybenzaldehyde. Activity of these enzymes was also demonstrated with ferulic acid to yield vanillin (Hirakawa et al. 2012), and therefore, we hypothesize that in mixtures containing coumaric acid and ferulic acid, CuoAB can transform both acids to their corresponding aldehydes (Fig. 1).

Since the degradation of $p$-coumaric acid by $R$. palustris has been demonstrated to occur via the indicated hypothetical reactions of this study. Compounds colored in blue ( $p$-coumaroyl-CoA and feruloyl-CoA) represent intermediates not measured

benzoyl-CoA pathway (Pan et al. 2008), we propose that $p$-hydroxybenzaldehyde is further transformed to $p$-hydroxybenzoic acid by an aldehyde dehydrogenase, in agreement to the pathway suggested by Pan et al. (2008). Then, $p$-hydroxybenzoic acid is activated by CoA ligation and enters the benzoyl-CoA pathway according to well established pathways (Egland et al. 1997; Harwood et al. 1998). On the other hand, ferulic acid has been shown to support photoheterotrophic growth of $R$. palustris even though this aromatic acid is only partially transformed to vanillic acid; the alkyl 
chain serves as the organic carbon source for growth (Harwood and Gibson 1988). Therefore, there is no evidence to suggest a potential pathway for the complete degradation of ferulic acid by $R$. palustris. Instead, we propose that ferulic acid is converted to vanillic acid via vanillin by CuoAB (Hirakawa et al. 2012) and an aldehyde dehydrogenase (Pan et al. 2008). In the experiments of Austin et al. (2015), vanillic acid accumulated as an extracellular product, but it is not known whether its accumulation represents the complete inability of $R$. palustris to degrade vanillic acid or a slow and incomplete degradation of this product. Experiments with vanillic acid as the sole carbon source show that this substrate does not support anaerobic growth of $R$. palustris (Harwood and Gibson 1988; Oshlag et al. 2020), and thus, if vanillic acid was degraded, we hypothesize that it occurred by cometabolism.

The hydrolysate used in the Austin et al. (2015) experiments also contained lower concentrations of the aromatic amides $p$-hydroxybenzamide, vanillamide, and syringamide. These aromatic substrates were partially degraded by $R$. palustris (Austin et al. 2015); thus, we hypothesize that they can be hydrolyzed by amidases to $p$-hydroxybenzoic acid, vanillic acid, and syringic acid, respectively (Fig. 1). Furthermore, by analogy to the transformation of $p$-hydroxybenzaldehyde and vanillin, we hypothesize that syringaldehyde is converted to syringic acid (Fig. 1), which accumulated extracellularly in the experiments of Austin et al. (2015). Syringic acid is not a substrate that supports anaerobic growth of wild type $R$. palustris. However, we recently adapted an $R$. palustris strain able to photoheterotrophically grow on this substrate as the sole organic carbon source (Oshlag et al. 2020). This adaptation took several rounds of selection that were not used in the experiments of Austin et al. (2015), in which syringic acid degradation was not apparent, and therefore, we hypothesize that syringic acid was not significantly co-metabolized in the experiments of Austin et al. (2015).

Three additional aromatic compounds were detected in the hydrolysates used by Austin et al. (2015), $p$-hydroxyacetophenone, acetovanillone, and acetosyringone. Of these compounds, the extracellular concentration of $p$-hydroxyacetophenone increased during growth in hydrolysates, whereas the concentrations of acetovanillone and acetosyringone remained constant throughout the study (Fig. S2).
Given that these compounds were found at low concentrations and that there is no evidence for their degradation by $R$. palustris, we did not include these substrates in the kinetic analysis described below.

Kinetic simulation of the metabolism of $\mathrm{p}$ hydroxyphenyl and guaiacyl type aromatics

We used the time series results presented in Austin et al. (2015) to evaluate whether the transformation pathways proposed in Fig. 1 were appropriate for simulating the simultaneous degradation of aromatic compounds by $R$. palustris and to elucidate factors that may influence their transformation rates. We first simulated each reaction step with a simple first order rate equation (see Materials and Methods), and then evaluated the impact of other assumptions on the goodness of fit between experimental results and model outputs (Table 2). Model outputs and optimized parameters are collectively shown in Fig. 2 and Table S1, and separately shown in the supplementary document (Fig. S3-S7). An assumption in these models is that the observed extracellular concentration of pathway intermediates provides a reasonable representation of the balance between the rates of production and degradation of each intermediate. This assumption was used for all intermediates that were extracellularly measurable. However, there was no measured concentration for the CoA-ligated intermediates, which only accumulate intracellularly, and therefore, the models assumed a hypothetical accumulation of these compounds.

The initial model, which used first order rates for all reactions (Case 1), resulted in good fits between predictions and most of the measured extracellular aromatics, but failed to predict the observed accumulation of $p$-hydroxybenzoic and vanillic acids (Fig. 2, Fig. S3). The fit of the model to the measured ferulic acid concentrations was deficient, likely because the concentrations of ferulic acid were much lower than the concentrations of other aromatics, and in general, the best-fitting technique will place less weight on matching simulated and experimental concentrations for this aromatic compound. The obtained best-fit reaction rates with this model (Table $\mathrm{S} 1$ ) indicated that the transformation rate for the CoA ligated intermediates was 2 to 3 orders of magnitude lower than the reaction rates of the upstream ( $p$-coumaric acid and ferulic acid) and the downstream ( $p$ - 


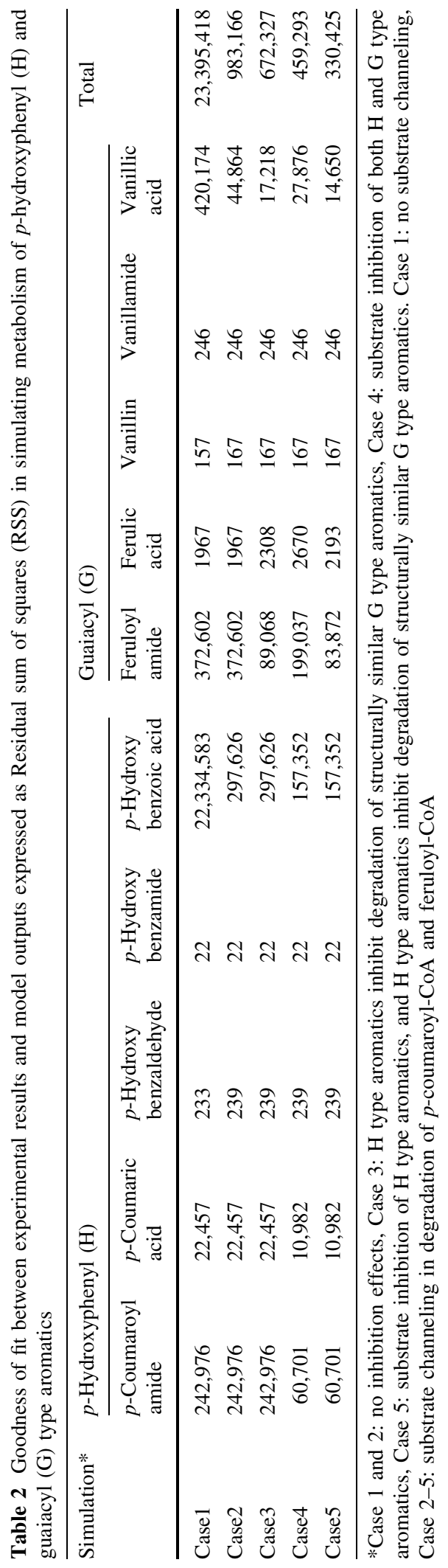




\section{A · p-Hydroxyphenyl}
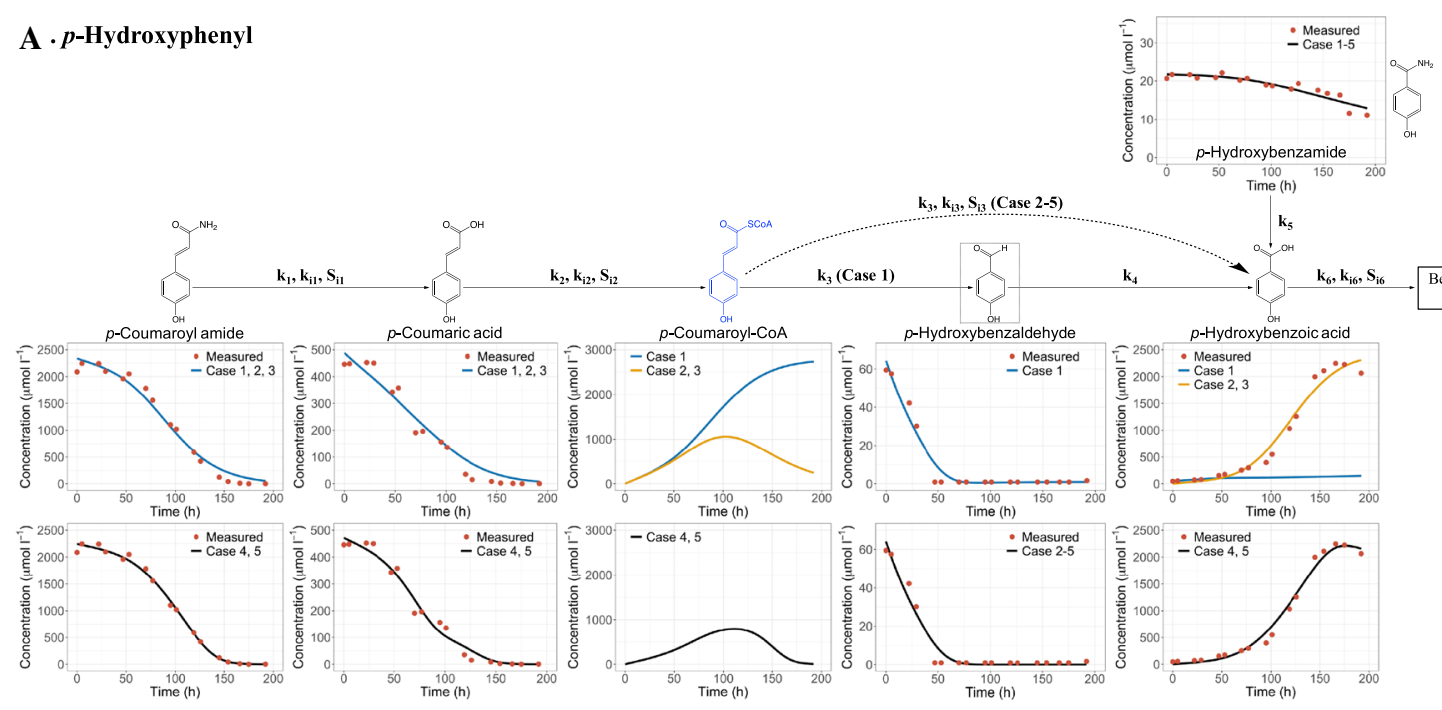

\section{B . Guaiacyl}
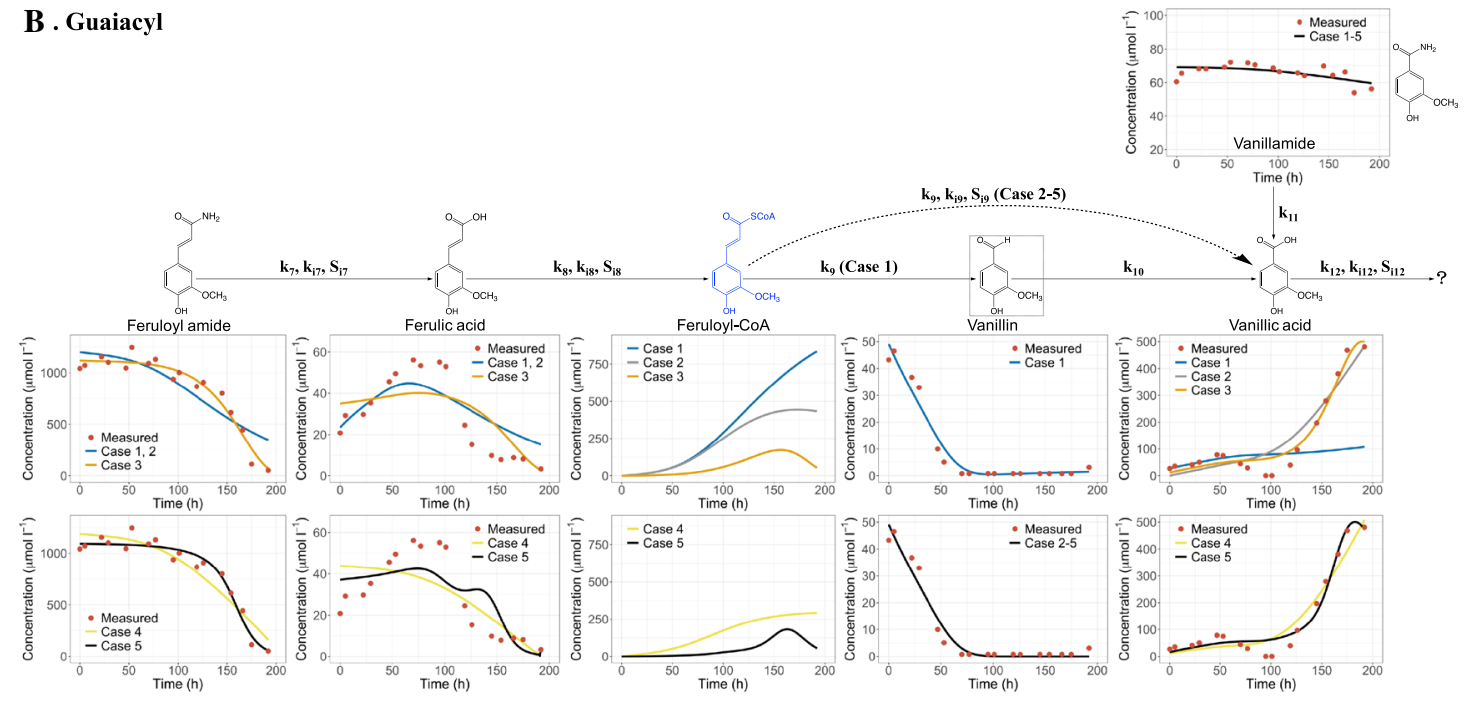

\section{Syringyl}

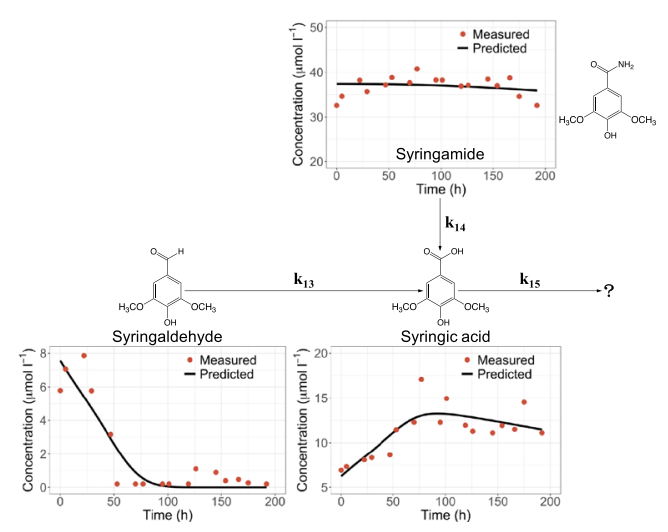

hydroxybenzaldehyde and vanillin) intermediates. Therefore, this simulation predicted a bottleneck in the pathways, with accumulation of $p$-coumaroyl-CoA and feruloyl-CoA, and since the model was not able to 
४Fig. 2 Kinetic modeling of a $p$-hydroxyphenyl, b guaiacyl, and c syringyl aromatics with alternative models exploring factors that may influence their transformation rates. For $p$-hydroxyphenyl and guaiacyl aromatics, Case 1 and 2: no inhibition effects; Case 3: $\mathrm{H}$ type aromatics inhibit degradation of structurally similar G type aromatics; Case 4: substrate inhibition of both $\mathrm{H}$ and $\mathrm{G}$ type aromatics; Case 5: substrate inhibition of $\mathrm{H}$ type aromatics, and $\mathrm{H}$ type aromatics inhibit degradation of structurally similar $\mathrm{G}$ type aromatics. Case 1: no substrate channeling; Case 2-5: substrate channeling in degradation of $p$-coumaroyl-CoA and feruloyl-CoA. Degradation rate $(\mathrm{k})$, inhibition factor $\left(\mathrm{k}_{\mathrm{i}}\right)$ and inhibitory substrate $\left(\mathrm{S}_{\mathrm{i}}\right)$ of each reaction are shown in Table $\mathrm{S} 1$

predict the accumulation of $p$-hydroxybenzoic and vanillic acids (Fig. 2), we interpreted these results as being an incorrect hypothetical accumulation of the CoA-ligated intermediates.

To improve the accuracy of this prediction, another simulation (Case 2) considered the direct transformation of $p$-coumaroyl-CoA and feruloyl-CoA to $p$ hydroxybenzoic and vanillic acid, respectively, without accumulation of $p$-hydroxybenzaldehyde or vanillin (Fig. 2, Fig. S4). This assumption is justified by experimental observations of little extracellular accumulation of vanillin during the transformation of ferulic acid to vanillic acid in other organisms, presumably due to rapid metabolism or substrate channeling to prevent the toxicity of the intermediate aldehyde (Fitzgerald et al. 2004; Priefert et al. 2001). Substrate channeling has been reported to occur in other microbial transformations in which the accumulation of toxic intermediates is minimized or prevented (Abernathy et al. 2017). With this modification, the model output (Fig. 2) accurately predicts the observed extracellular accumulation of $p$-hydroxybenzoic and vanillic acids. Compared to the simulation of Case 1, the revised model predicts accumulation and degradation of the CoA ligated intermediates and rate constants for these steps that are of similar order of magnitude as other reactions in the pathway (Table S1). Because of the improvement observed in Case 2, substrate channeling in degradation of $p$ coumaroyl-CoA and feruloyl-CoA was assumed in all subsequent refinements of the model (Fig. 2, Fig. S5S7).

By comparing Case 2 simulation results with the experimental observations of $\mathrm{G}$ type aromatics metabolism, we noted an apparent lag in the experimental degradation of feruloyl amide that was not captured by the model (Fig. 2). Since there is experimental evidence that the CuoAB enzymes, responsible for the transformations from $p$-coumaric acid to $p$-hydroxybenzaldehyde, are active on the transformation of ferulic acid to vanillin (Hirakawa et al. 2012), we simulated a situation in which the metabolism of the $\mathrm{G}$ type aromatics is inhibited by the binding of these $\mathrm{H}$ type aromatics to these enzymes. That is, it may be possible that $\mathrm{G}$ aromatics can bind to these H-pathway enzymes at a lower affinity due to the presence of the meta-methoxy substitution on the ring. Therefore, we modified the kinetic expressions in the modeling of the degradation of feruloyl amide, ferulic acid and feruloyl-CoA by simulating competitive inhibition (See Materials and Methods) by $p$-coumaroyl amide, $p$-coumaric acid, and $p$-coumaroyl-CoA, respectively (Case 3; Fig. 2, Fig. S5).

By allowing competitive inhibition of $G$ type aromatics by the corresponding $\mathrm{H}$ type aromatics, the simulation in Case 3 greatly improved the fit of experiments to simulations, specifically for feruloyl amide and vanillic acid (Table 2). The accumulation of vanillic acid in the Case 2 simulation (Fig. 2) was accompanied by a prediction that this aromatic was not degraded (i.e., the degradation rate was zero; Table S1). This is consistent with previous reports that this aromatic cannot be metabolized by wild type R. palustris anaerobically (Harwood and Gibson 1988; Oshlag et al. 2020). However, in the Case 3 simulation, the best-fit solution required vanillic acid to have a non-zero degradation rate (Table S1), suggesting cometabolism of vanillic acid in the aromatic mixture. Since a pathway for vanillic acid degradation cannot be inferred from the available data, we used the simple first order rate to describe vanillic acid degradation instead of assuming competitive inhibition by $p$ hydroxybenzoic acid. The same description was also applied in modeling vanillic acid degradation in subsequent cases.

While the simulation of Case 3 provided a good fit to the experimental trends, experiments and simulations of $p$-coumaroyl amide and $p$-coumaric acid diverged after $100 \mathrm{~h}$, with experimental observations showing a faster degradation rate than predicted by this version of the model (Fig. 2). To further attempt to improve the match of simulations to experiments, we explored the hypothesis of substrate inhibition. That is, we modified the kinetic expression so that the aromatic compound degradation was inhibited at high substrate concentrations (see Materials and Methods). 
This modification was implemented for $p$-coumaroyl amide, $p$-coumaric acid, $p$-coumaroyl-CoA, and $p$ hydroxybenzoic acid belonging to $\mathrm{H}$ type aromatics, and feruloyl amide, ferulic acid, feruloyl-CoA and vanillic acid for the $\mathrm{G}$ type aromatics. This instance of the model (Case 4; Fig. 2, Fig. S6) best fitted the experimental results of each $\mathrm{H}$ type aromatics, but the fit to $\mathrm{G}$ type aromatics was generally poorer than in Case 3 (Table 2).

By comparing the assumptions in Cases 2-4, a hybrid of kinetic expressions was simulated, in which $\mathrm{H}$ type aromatics were dominantly impacted by substrate inhibition (as simulated in Case 4), and G type aromatics were impacted by competitive inhibition (as simulated in Case 3). This instance of the model (Case 5; Fig. 2, Fig S7) best fitted experimental results of both $\mathrm{H}$ and $\mathrm{G}$ type aromatics compared with previous cases (Table 2).

Simulation of vanillic acid and benzoic acid metabolism

Overall, our simulations supported the hypothesis that vanillic acid is consumed in the experiments. Although no vanillic acid degradation was simulated by Cases 1 and 2, a positive degradation rate was predicted as we refined the simulations in Cases 3-5 (Table S1). Vanillic acid is known not to be degraded anaerobically by $R$. palustris as sole organic carbon source (Harwood and Gibson 1988; Oshlag et al. 2020). Therefore, we propose that vanillic acid was co-metabolized through a pathway that required the presence of other aromatics, as has been observed previously in this bacterium (Gall et al. 2013). Current knowledge on metabolism of vanillic acid by other bacteria involves demethylation and conversion to protocatechuic acid either by vanillate demethylase (VanA and VanB) which is reported to require oxygen (Chen et al. 2012; Priefert et al. 1997; Segura et al. 1999; Sudtachat et al. 2009; Venturi et al. 1998) or $\mathrm{H}_{4}$ folate-dependent aromatic O-demethylases which can be active under anaerobic conditions (Abe et al. 2005; Berman and Frazer 1992; Kaufmann et al. 1998; Naidu and Ragsdale 2001; Nishikawa et al. 1998). We found that simulating the production of protocatechuic acid from vanillic acid did not yield a good fit to the observed extracellular levels of protocatechuic acid (Fig. 3a). Therefore, it is possible that the experimentally observed protocatechuic acid accumulation is not due to vanillic acid degradation, but to the degradation of another aromatic compound in the hydrolysate medium that was not identified.

It is also known that benzoic acid, $p$-hydroxybenzoic acid and protocatechuic acid are all degraded through benzoyl-CoA pathway in R. palustris (Egland et al. 1997; Gall et al. 2013; Harwood et al. 1998). The experimental results of Austin et al. (2015) showed extracellular accumulation of both $p$-hydroxybenzoic acid and protocatechuic acid. The benzoic acid concentration remained relatively constant, and a first-order degradation model suggested a relatively low degradation rate (Fig. 3b). The accumulation of $p$ hydroxybenzoic acid (Fig. 2) and the low degradation rate of benzoic acid compared with other $\mathrm{H}$ type aromatics (Fig. 3b) indicated that, in the experiments of results of Austin et al. (2015), substrates entering the benzoyl-CoA pathway was a limiting step in the degradation of the aromatic mixtures by $R$. palustris.

Kinetic simulation of the metabolism of syringyl aromatics

Syringyl type aromatics with longer alkyl chain such as sinapoyl amide or sinapic acid were not detected in these biomass hydrolysates (Table 1, Fig. S1), and therefore, the kinetic modeling of $\mathrm{S}$ aromatics was limited to syringamide, syringaldehyde, and syringic acid. A simulation using first order rates for the degradation of these three $\mathrm{S}$ aromatics (Fig. 2) showed best fits when there were positive degradation rates for the three metabolites (Table S1). Given the adequate fits observed with the first order rates and the low concentrations of these metabolites, this was the only model used for the $\mathrm{S}$ type aromatics. Comparing the transformation rates of the aromatic aldehydes (Table S1), the degradation rates follow the trend of $p$-hydroxybenzaldehyde $>$ vanillin $>$ syringaldehyde. Similarly, the transformation rates of aromatic amides followed the trend of $p$-hydroxybenzamide $>$ vanillamide $>$ syringamide (Table S1). Thus, the kinetic simulations do suggest that a higher number of methoxy substitutions decreases the rate at which these compounds are metabolized. Similar to vanillic acid, syringic acid is known not to be degraded anaerobically by $R$. palustris as sole organic carbon source (Harwood and Gibson 1988). Degradation of syringic acid by other bacteria is usually initiated by demethylation, via demethylase enzymes that 


\section{A. Protocatechuic acid}

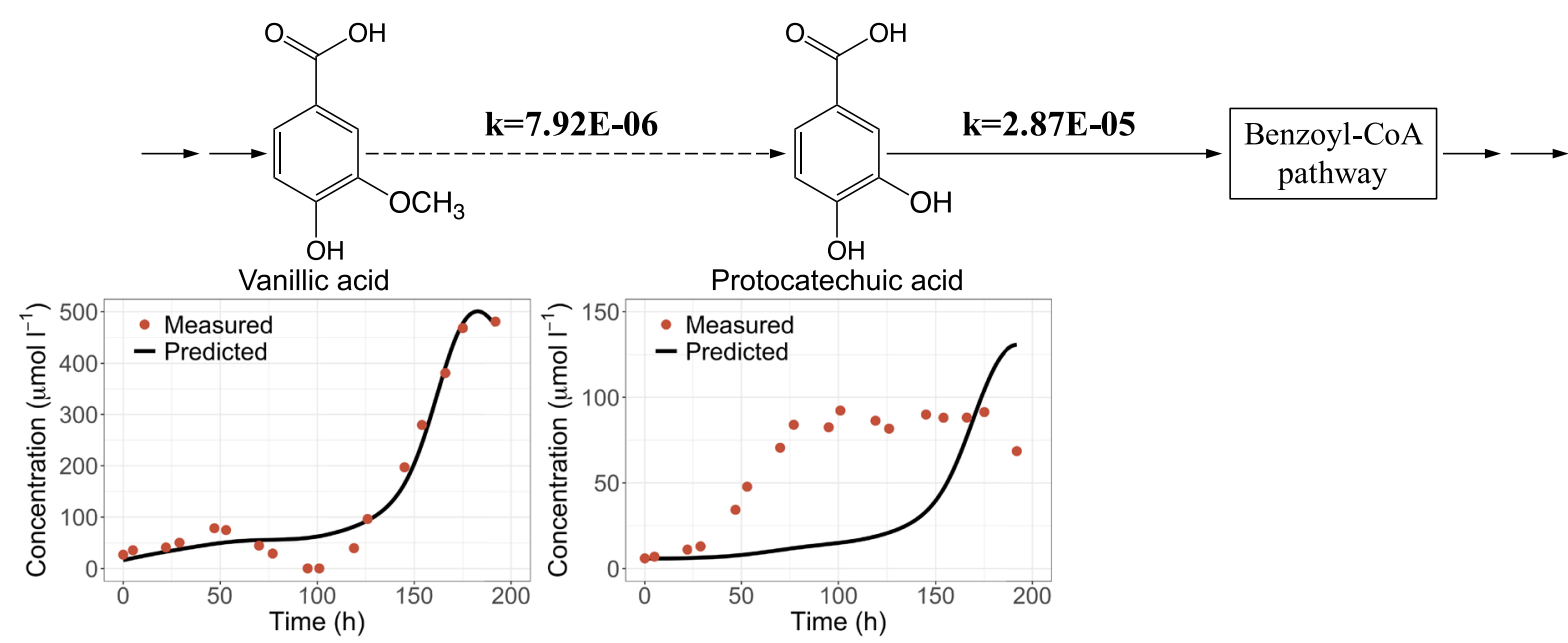

\section{B. Benzoic acid}

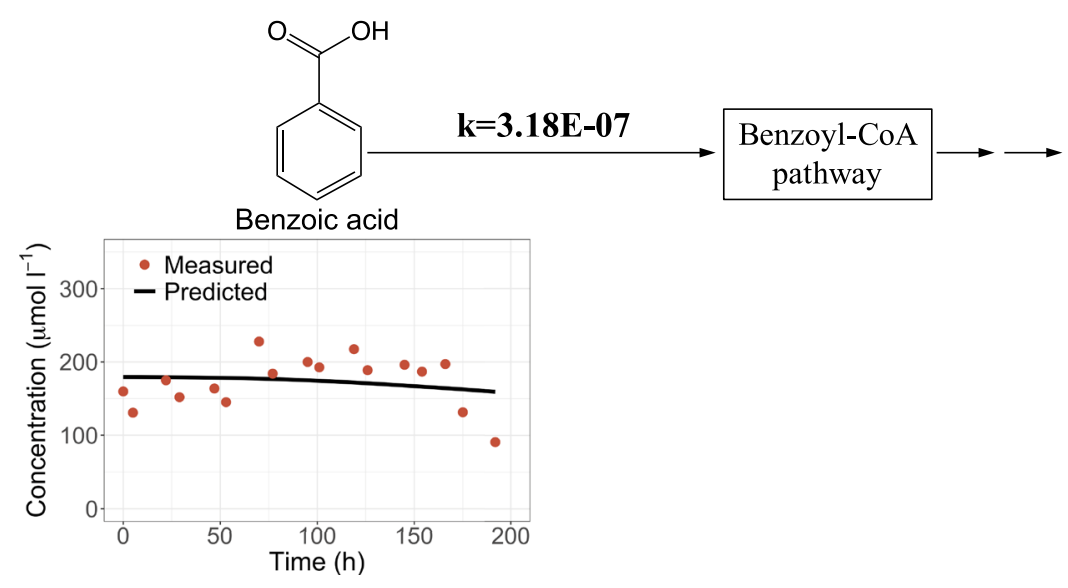

Fig. 3 Comparison of experimental and modeling results for a protocatechuic acid production from vanillic acid assuming first order rates of protocatechuic production and consumption,

typically do not require oxygen as a substrate (Abe et al. 2005; Kasai et al. 2005; Masai et al. 2004; Wu et al. 1988). Using first order degradation rates, our model predicts a small positive rate for syringic acid degradation (Table S1). Although syringyl compounds are found in low concentration in these hydrolysates, this finding can be used to propose that, contrary to our initial hypothesis, syringic acid may be co-metabolized in the aromatic hydrolysate mixture. However, further study is needed to elucidate whether the syringic acid was metabolized via the benzoyl-CoA pathway or other unknown pathways, as suggested by and $\mathbf{b}$ benzoic acid consumption assuming first order degradation rate. The units of the estimated first order rates are 1 $\mathrm{mg}^{-1} \mathrm{~h}^{-1}$

analysis of an evolved strain of $R$. palustris that acquired the ability to use this aromatic acid as a sole carbon source (Oshlag et al. 2020).

Concluding remarks

The kinetics-based simulation analysis presented here offers predictions for co-metabolism of several aromatic compounds when present in complex mixtures. Although various models have been applied to describe kinetics of co-metabolic degradations (Alvarez-Cohen and Speitel 2001; Wang et al. 
2002, 2014), the first order kinetic and rates that account for inhibition by a secondary substrate described in this study provided an applicable method to simulate systems including complex pathways and inhibition effects. Overall, the proposed $p$-hydroxyphenyl, guaiacyl and syringyl degradation pathways (Fig. 1) were supported by the fit between experimental results and simulation outputs. Furthermore, the kinetic analysis suggested that co-metabolism of aromatic compounds may be impacted by substrate inhibition and competitive inhibition, presumably because the ability of $R$. palustris to degrade multiple aromatic compounds is based on having enzymes with broad substrate specificity. Substrate inhibition can impact degradation of $\mathrm{H}$ type aromatics, whereas degradation of $\mathrm{G}$ type aromatics can be impacted by inhibition from competing $\mathrm{H}$ type aromatics. These models make several new predictions for experimental studies to provide a more thorough understanding of the pathways and factors influencing degradation of aromatics by $R$. palustris. Ultimately, the results of future experimental and modeling studies will contribute to furthering the knowledge of aromatic metabolism by $R$. palustris that could enable engineering approaches that improve the ability of this and other bacteria to convert plant-derived aromatic mixtures into valuable products.

Acknowledgements This study was based upon work supported in part by the Great Lakes Bioenergy Research Center, U.S. Department of Energy, Office of Science, Office of Biological and Environmental Research under Award Number DE-SC0018409 and the National Science Foundation (Grant CBET-1506820).

Funding This study was based upon work supported in part by the Great Lakes Bioenergy Research Center, U.S. Department of Energy, Office of Science, Office of Biological and Environmental Research under Award Number DESC0018409 and the National Science Foundation (Grant CBET-1506820).

Data availability The authors confirm that the data supporting the findings of this study are available within the article and its supplementary materials.

\section{Compliance with ethical standards}

Conflict of interest The authors declare that they have no conflict of interest.

Open Access This article is licensed under a Creative Commons Attribution 4.0 International License, which permits use, sharing, adaptation, distribution and reproduction in any medium or format, as long as you give appropriate credit to the original author(s) and the source, provide a link to the Creative Commons licence, and indicate if changes were made. The images or other third party material in this article are included in the article's Creative Commons licence, unless indicated otherwise in a credit line to the material. If material is not included in the article's Creative Commons licence and your intended use is not permitted by statutory regulation or exceeds the permitted use, you will need to obtain permission directly from the copyright holder. To view a copy of this licence, visit http://creativecommons.org/licenses/by/4.0/.

\section{References}

Abe T, Masai E, Miyauchi K, Katayama Y, Fukuda M (2005) A tetrahydrofolate-dependent O-demethylase, LigM, is crucial for catabolism of vanillate and syringate in Sphingomonas paucimobilis SYK-6. J Bacteriol 187:2030-2037. https://doi.org/10.1128/Jb.187.6.2030-2037.2005

Abernathy MH, He L, Tang YJ (2017) Channeling in native microbial pathways: implications and challenges for metabolic engineering. Biotechnol Adv 35:805-814. https://doi.org/10.1016/j.biotechadv.2017.06.004

Alvarez-Cohen L, Speitel GE (2001) Kinetics of aerobic cometabolism of chlorinated solvents. Biodegradation 12:105-126. https://doi.org/10.1023/A:1012075322466

Atkinson KA (1989) An Introduction to numerical analysis, 2nd edn. John Wiley and Sons, New York

Austin S et al (2015) Metabolism of multiple aromatic compounds in corn stover hydrolysate by Rhodopseudomonas palustris. Environ Sci Technol 49:8914-8922. https://doi. org/10.1021/acs.est.5b02062

Berman MH, Frazer AC (1992) Importance of tetrahydrofolate and ATP in the anaerobic O-demethylation reaction for phenylmethylethers. Appl Environ Microb 58:925-931

Chen HP, Chow M, Liu CC, Lau A, Liu J, Eltis LD (2012) Vanillin catabolism in Rhodococcus jostii RHA1. Appl Environ Microb 78:586-588. https://doi.org/10.1128/Aem. 06876-11

Davis KM, Rover M, Brown RC, Bai XL, Wen ZY, Jarboe LR (2016) Recovery and utilization of lignin monomers as part of the biorefinery approach. Energies. https://doi.org/10. 3390/en9100808

Egland PG, Pelletier DA, Dispensa M, Gibson J, Harwood CS (1997) A cluster of bacterial genes for anaerobic benzene ring biodegradation. Proc Natl Acad Sci USA 94:6484-6489. https://doi.org/10.1073/pnas.94.12.6484

Elder DJE, Morgan P, Kelly DJ (1992) Anaerobic degradation of trans-cinnamate and omega-phenylalkane carboxylicacids by the photosynthetic bacterium rhodopseudomonaspalustris - evidence for a beta-oxidation mechanism. Arch Microbiol 157:148-154. https://doi.org/10.1007/ Bf00245283

Fitzgerald DJ, Stratford M, Gasson MJ, Ueckert J, Bos A, Narbad A (2004) Mode of antimicrobial action of vanillin against Escherichia coli, Lactobacillus plantarum and 
Listeria innocua. J Appl Microbiol 97:104-113. https://doi. org/10.1111/j.1365-2672.2004.02275.x

Gall DL, Ralph J, Donohue TJ, Noguera DR (2013) Benzoyl coenzyme a pathway-mediated metabolism of meta-hydroxy-aromatic acids in Rhodopseudomonas palustris. J Bacteriol 195:4112-4120. https://doi.org/10.1128/Jb. 00634-13

Gall DL, Ralph J, Donohue TJ, Noguera DR (2017) Biochemical transformation of lignin for deriving valued commodities from lignocellulose. Curr Opin Biotech 45:120-126. https://doi.org/10.1016/j.copbio.2017.02.015

Gompertz B (1825) On the nature of the function expressive of the law of human mortality, and on a new mode of determining the value of life contingencies. Phil Trans R Soc B 115:513-583

Govindaraju A, McKinlay JB, LaSarre B (2019) Phototrophic lactate utilization by Rhodopseudomonas palustris is stimulated by coutilization with additional substrates. Appl Environ Microb. https://doi.org/10.1128/AEM.00048-19

Harwood CS, Burchhardt G, Herrmann H, Fuchs G (1998) Anaerobic metabolism of aromatic compounds via the benzoyl-CoA pathway. Fems Microbiol Rev 22:439-458. https://doi.org/10.1016/S0168-6445(98)00026-6

Harwood CS, Gibson J (1988) Anaerobic and aerobic metabolism of diverse aromatic compounds by the photosynthetic bacterium Rhodopseudomonas palustris. Appl Environ Microb 54:712-717

Hirakawa H, Schaefer AL, Greenberg EP, Harwood CS (2012) Anaerobic $p$-coumarate degradation by Rhodopseudomonas palustris and identification of CouR, a MarR repressor protein that binds $p$-coumaroyl coenzyme A. J Bacteriol 194:1960-1967. https://doi.org/10.1128/Jb. 06817-11

Hirrlinger B, Stolz A, Knackmuss HJ (1996) Purification and properties of an amidase from Rhodococcus erythropolis MP50 which enantioselectively hydrolyzes 2-arylpropionamides. J Bacteriol 178:3501-3507. https://doi.org/10. 1128/jb.178.12.3501-3507.1996

Ismailsab M, Monisha TR, Reddy PV, Santoshkumar M, Nayak AS, Karegoudar TB (2017) Biotransformation of aromatic and heterocyclic amides by amidase of whole cells of Rhodococcus sp. MTB5: biocatalytic characterization and substrate specificity. Biocatal Biotransfor 35:74-85. https://doi.org/10.1080/10242422.2017.1282467

Kasai D, Masai E, Miyauchi K, Katayama Y, Fukuda M (2005) Characterization of the gallate dioxygenase gene: three distinct ring cleavage dioxygenases are involved in syringate degradation by Sphingomonas paucimobilis SYK-6. J Bacteriol 187:5067-5074. https://doi.org/10.1128/Jb. 187.15.5067-5074.2005

Kaufmann F, Wohlfarth G, Diekert G (1998) O-demethylase from Acetobacterium dehalogenans substrate specificity and function of the participating proteins. Eur J Biochem 253:706-711. https://doi.org/10.1046/j.1432-1327.1998. 2530706.x

Masai E et al (2004) A novel tetrahydrofolate-dependent $O$ demethylase gene is essential for growth of Sphingomonas paucimobilis SYK-6 with syringate. J Bacteriol 186:2757-2765. https://doi.org/10.1128/Jb.186.9.27572765.2004
Naidu D, Ragsdale SW (2001) Characterization of a threecomponent vanillate $O$-demethylase from Moorella thermoacetica. J Bacteriol 183:3276-3281. https://doi.org/10. 1128/Jb.183.11.3276-3281.2001

Nishikawa S et al (1998) Cloning and sequencing of the Sphingomonas (Pseudomonas) paucimobilis gene essential for the $\mathrm{O}$ demethylation of vanillate and syringate. Appl Environ Microb 64:836-842

Oshlag JZ et al (2020) Anaerobic degradation of syringic acid by an adapted strain of Rhodopseudomonas palustris. Appl Environ Microb. https://doi.org/10.1128/AEM.01888-19

Pan C et al (2008) Characterization of anaerobic catabolism of p-coumarate in Rhodopseudomonas palustris by integrating transcriptomics and quantitative proteomics. Mol Cell Proteomics 7:938-948. https://doi.org/10.1074/mcp. M700147-MCP200

Perez JM et al (2019) Funneling aromatic products of chemically depolymerized lignin into 2-pyrone-4-6-dicarboxylic acid with Novosphingobium aromaticivorans. Green Chem 21:1340-1350. https://doi.org/10.1039/c8gc03504k

Priefert H, Rabenhorst J, Steinbuchel A (2001) Biotechnological production of vanillin. Appl Microbiol Biot 56:296-314. https://doi.org/10.1007/s002530100687

Priefert H, Rabenhorst J, Steinbüchel A (1997) Molecular characterization of genes of Pseudomonas sp. strain HR199 involved in bioconversion of vanillin to protocatechuate. J Bacteriol 179:2595-2607. https://doi.org/10. 1128/jb.179.8.2595-2607.1997

R Core Team (2019) R: a language and environment for statistical computing. R Foundation for Statistical Computing, Vienna, Austria

Ragauskas AJ et al (2014) Lignin valorization: improving lignin processing in the biorefinery. Science. https://doi.org/10. 1126/science. 1246843

Ruan LT, Zheng RC, Zheng YG (2016) Mining and characterization of two amidase signature family amidases from Brevibacterium epidermidis ZJB-07021 by an efficient genome mining approach. Protein Expres Purif 126:16-25. https://doi.org/10.1016/j.pep.2016.05.006

Segura A, Bünz PV, D’Argenio DA, Ornston LN (1999) Genetic analysis of a chromosomal region containing vanA and $v a n B$, genes required for conversion of either ferulate or vanillate to protocatechuate in Acinetobacter. J Bacteriol 181:3494-3504

Sudtachat N et al (2009) Aerobic vanillate degradation and c-1 compound metabolism in Bradyrhizobium japonicum. Appl Environ Microb 75:5012-5017. https://doi.org/10. 1128/Aem.00755-09

Teymouri F, Laureano-Perez L, Alizadeh H, Dale BE (2004) Ammonia fiber explosion treatment of corn stover. Appl Biochem Biotech 113:951-963

Tuck CO, Pérez E, Horváth IT, Sheldon RA, Poliakoff M (2012) Valorization of biomass: deriving more value from waste. Science 337:695-699. https://doi.org/10.1126/science. 1218930

Venturi V, Zennaro F, Degrassi G, Okeke BC, Bruschi CV (1998) Genetics of ferulic acid bioconversion to protocatechuic acid in plant-growth-promoting Pseudomonas putida WCS358. Microbiol-Sgm 144:965-973. https://doi. org/10.1099/00221287-144-4-965 
Wang JL, Quan XC, Han LP, Qian Y, Hegemann W (2002) Kinetics of co-metabolism of quinoline and glucose by Burkholderia pickettii. Process Biochem 37:831-836

Wang SZ, Yang Q, Zhang LN, Wang YY (2014) Kinetics of the aerobic co-metabolism of 1,1-dichloroethylene by Achromobacter sp.: a novel benzene-grown culture. Biotechnol Lett 36:1271-1278. https://doi.org/10.1007/s10529-0141490-9

Wong DWS (2009) Structure and action mechanism of ligninolytic enzymes. Appl Biochem Biotech 157:174-209. https://doi.org/10.1007/s12010-008-8279-z
Wu ZG, Daniel SL, Drake HL (1988) Characterization of a codependent O-demethylating enzyme-system from the acetogen Clostridium thermoaceticum. J Bacteriol 170:5747-5750. https://doi.org/10.1128/jb.170.12.57475750.1988

Publisher's Note Springer Nature remains neutral with regard to jurisdictional claims in published maps and institutional affiliations. 